\title{
Lumbosacral plexus injury following vaginal delivery with epidural analgesia -A case report-
}

\author{
Seil Park, Sung Wook Park, and Keon Sik Kim \\ Department of Anesthesiology and Pain Medicine, Kyung Hee University College of Medicine, Seoul, Korea
}

A 26 year old, healthy, 41 week primiparous woman received a patient-controlled epidural analgesia (PCEA) and experienced paraplegia 11 hours later after a vaginal delivery. This was thought to be the result of complications from PCEA but there was no specific abnormality on magnetic resonance imaging (MRI) of the lumbosacral spine. On an electromyography (EMG) study performed 15 days following delivery, signs of tibial neuropathy were present and peripheral nerve injury during vaginal delivery was suspected. Motor weakness and hypoesthesia of both lower extremities improved rapidly, but a decrease in the desire to urinate or defecate, followed by urinary incontinence and constipation persisted, We suspected the sacral plexus had been severely damaged during vaginal delivery. Seven months later, the patient's conditions improved but had not fully recovered. (Korean J Anesthesiol 2013; 64: 175-179)

Key Words: Analgesia, Epidural, Lumbosacral plexus, Obstetric delivery.

Neuropathy during vaginal delivery can be caused by a mother's preexisting neurologic impairment, manipulation of instruments during the delivery process, lithotomy position, compression by the head of the fetus, anesthetic procedures and a combination of other causes, but occasionally the cause can be idiopathic [1]. Even though neurological complications in obstetric patients after regional anesthesia are rare, having other causes in most cases, often the anesthesia process is first to be suspected, much to the chagrin of anesthesiologists.

We have experienced neurological complications in a patient after birth that was given PCEA. Through there was no specific abnormality on neurologic examination and other diagnostic studies, we concluded that the sacral plexus was found to be damaged during delivery.

\section{Case Report}

A primiparous 26 year old mother, 41 weeks of gestational age wanted a vaginal delivery. She had no specific abnormalities on medical history and in prenatal tests coagulation profiles.

Received: March 19, 2012. Revised: 1st, April 16, 2012; 2nd, April 18, 2012; 3rd, April 23, 2012. Accepted: April $26,2012$.

Corresponding author: Keon Sik Kim, M.D., Department of Anesthesiology and Pain Medicine, Kyung Hee University Medical Center, Hoegidong, Dongdaemun-gu, Seoul 130-702, Korea. Tel: 82-2-958-8591, Fax: 82-2-958-8580, E-mail: keonsik@hanafos.com (c) This is an open-access article distributed under the terms of the Creative Commons Attribution Non-Commercial License (http:// creativecommons.org/licenses/by-nc/3.0/), which permits unrestricted non-commercial use, distribution, and reproduction in any medium, provided the original work is properly cited. 

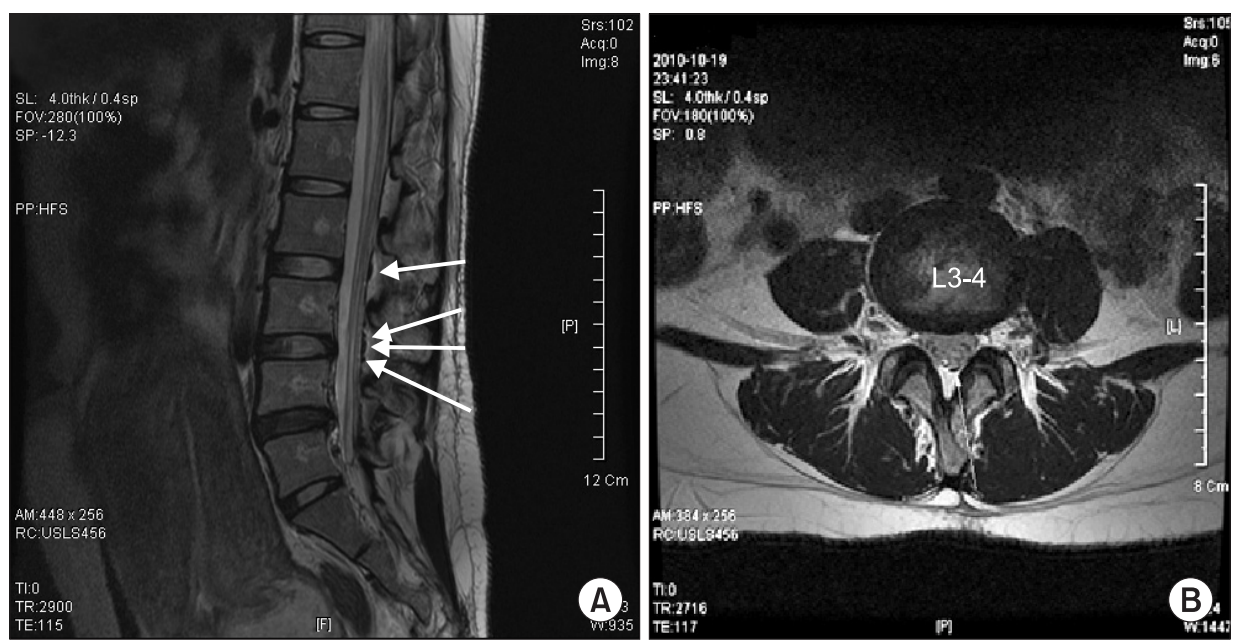

Fig. 1. Lumbosacral MRI (Postpartum 0 day). Sagittal (A) and axial (B) images of T2-weighted lumbosacral MRI show L3 through L5 intervertebral discs protrusion (central to left central) and associated air bubbles (white arrows), posterior epidural space - related to previous epidural catheterization.

To provide analgesia during the delivery, an epidural catheter was inserted in the L4-L5 interspace using the loss-ofresistance technique. No cerebrospinal fluid or blood leakage was observed and the patient did not experience paresthesia of the lower extremity, numbness, or pain. At the time $0.15 \%$ levobupivacaine $9 \mathrm{mg}$ and fentanyl $50 \mathrm{ug}$ was administered, the cervical os was dilated to about $2 \mathrm{~cm}$, effaced $100 \%$, and labor pains were 1 to 2 minutes apart with a visual analogue scale of 4 to 5 points. Nothing unusual was observed during the administration of the drugs, so a mixture of $97 \mathrm{ml}$ of $0.125 \%$ levobupivacaine and $150 \mathrm{ug}$ of fentanyl was administered at a $10 \mathrm{ml} / \mathrm{h}$ flow rate via the epidural space using the PCA infusion pump (AutoMed $3300^{\circledR}$, ACE-Medical, Seoul, Korea). The PCEA was programmed with a background infusion mode without bolus or lockout time. The mother gave birth to a $3.03 \mathrm{~kg}$ male newborn baby without issue other than the use of the vacuum. Stage 2 of labor was $78 \mathrm{~min}$. The newborn's 1 min Apgar score was 6 points. The neonate did not cry, had pale skin, and had no response to stimulation. As the Apgar score did not improve, the child was immediately moved to the neonatal intensive care unit. 80 minutes after birth the epidural catheter was removed. The total amount of drugs administered to that specified point, totaled $82 \mathrm{ml}$ of the $0.125 \% 97 \mathrm{ml}$ of levobupivacaine and $150 \mathrm{ug}$ of fentanyl mixture during $82 \mathrm{~min}$.

The patient experienced no ambulatory or urinary discomfort immediately post delivery but 11 hours afterward the patient reported hypoesthesia, numbness, tingling, and muscle weakness in both lower extremities enough to have trouble standing. Cauda equina syndrome caused by an epidural hematoma was suspected so a lumbar spine X-ray and lumbosacral MRI were taken, the MRI results only showed a L3 through L5 intervertebral disc protrusion (Fig. 1). The patient reported the same symptoms the next day as well as trouble detecting the desire to urinate and defecate. On postpartum day
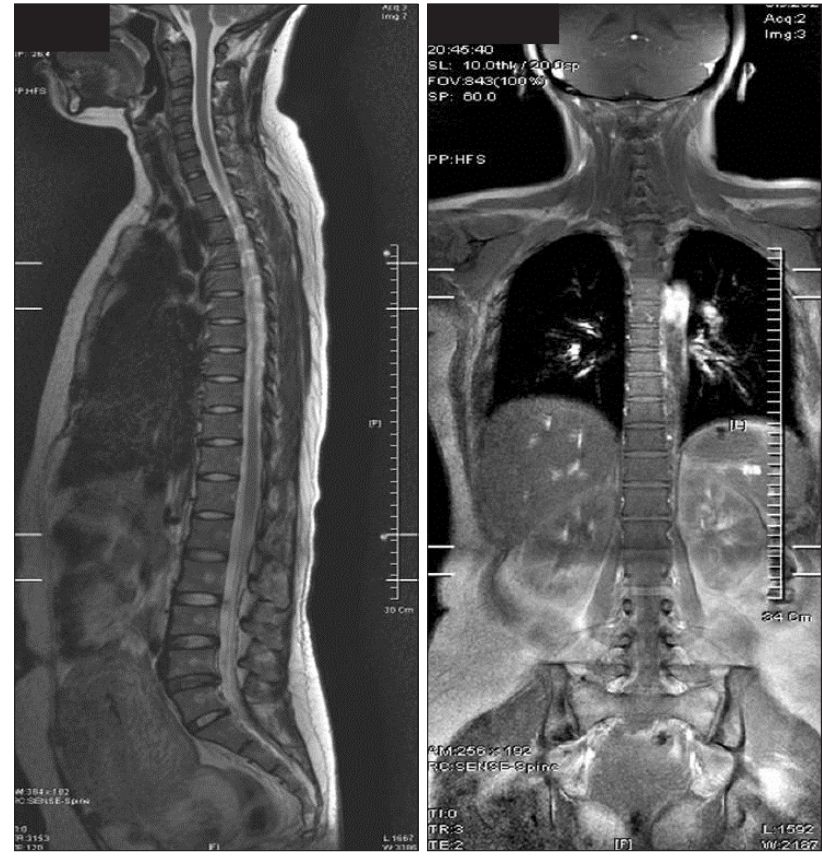

Fig. 2. Whole spine MRI (Postpartum 2 day). Whole spine MRI shows L3 through L5 intervertebral discs protrusion (central to left central), intervertebral disc bulging from $\mathrm{C} 6$ to $\mathrm{C} 7$, and associated air bubbles, posterior epidural space - related to previous epidural catheterization.

2, after consulting a neurologist, myelopathy was suspected so a whole spine MRI was taken but no abnormalities were found (Fig. 2). On postpartum day 4, strength in the lower extremities recovered enough to walk with support and limited hip, back of the thigh, calves and feet sensations returned. However, the patient still had trouble detecting the need to urinate/defecate and urinary retention, incontinence and constipation persisted. 15 days following delivery, an EMG showed that the motor nerve conduction by both tibial nerves were normal but right- 
sided compound muscle action potential was reduced. While Hoffmann's reflex on the left-side was not undulated in terms of tibial nerve damage, peripheral neuropathy was suspected.

After improvement in sensory nerve function, the patient left by her own volition to follow-up at another facility. 21 days after delivery, neurological observation revealed: hip flexion/extension was $4+/ 4$ on the right, $4-/ 4$ on the left; $4+/ 4$ for right hip abduction/adduction, 4-/4 for the left; $4+/ 4+$ for both left and right knee flexion/extension; ankle dorsiflexion/ plantarflexion 5/5 for left and right; , and big toe dorsiflexion/ plantarflexion $5 / 5$ for left and right, stable enough to walk without discomfort. But perianal area sensations and the lateral side of calf was both $60 / 60$, and the urination/defecation problem did not improve. Consequently the patient was rehospitalized and transferred to the department of neurology. The neurologist had an impression that the lumbosacral plexus was injured so steroid pulse therapy with $500 \mathrm{mg}$ of methyl prednisolone was started and further tests were scheduled.

The urodynamic study results showed bladder filling, a pressure of $6 \mathrm{mmH}_{2} \mathrm{O}$ (normal value $<10 \mathrm{mmH}_{2} \mathrm{O}$ ) and normal bladder compliance and capacity. But the first desire to void bladder volume was $373 \mathrm{ml}$ (normal value 175-250 ml) and the normal desire to void bladder volume was $591 \mathrm{ml}$ (normal value $350-450 \mathrm{ml}$ ), while the patient could not detect a strong desire to void. During urination, self-voiding urine was $10 \mathrm{ml}$ and residual urine was $600 \mathrm{ml}$, allowing for a final diagnosis of flaccid neurogenic bladder with a severely reduced contractility of bladder sensation and detrusor muscle.

Impaired rectal sensation was concluded with anorectal manometry results at rectal sensory threshold volume for first desire to defecate measured as $30 \mathrm{ml}$ (normal value 17-23 $\mathrm{ml}$ ), anal pressure reduced to $32 \mathrm{mmHg}$ (normal value $59-74$ $\mathrm{mmHg}$ ) at resting, and $12 \mathrm{mmHg}$ (normal value $80-160 \mathrm{mmHg}$ ) while squeezing. In terms of defecography, the anorectal angle, which is deeply related to the pudendal nerve, was measured (Fig. 3). The anorectal angle decreased when squeezing, however not enough to reach normal values. Also, the angle was 123 degrees at straining and 126 degrees at defecating which was higher than normal values, and a tendency for proctocolic intussusceptions was observed when defecating, showing a problem in contraction and relaxation. Pudendal nerve terminal motor latency (PNTML) was also planned but was not
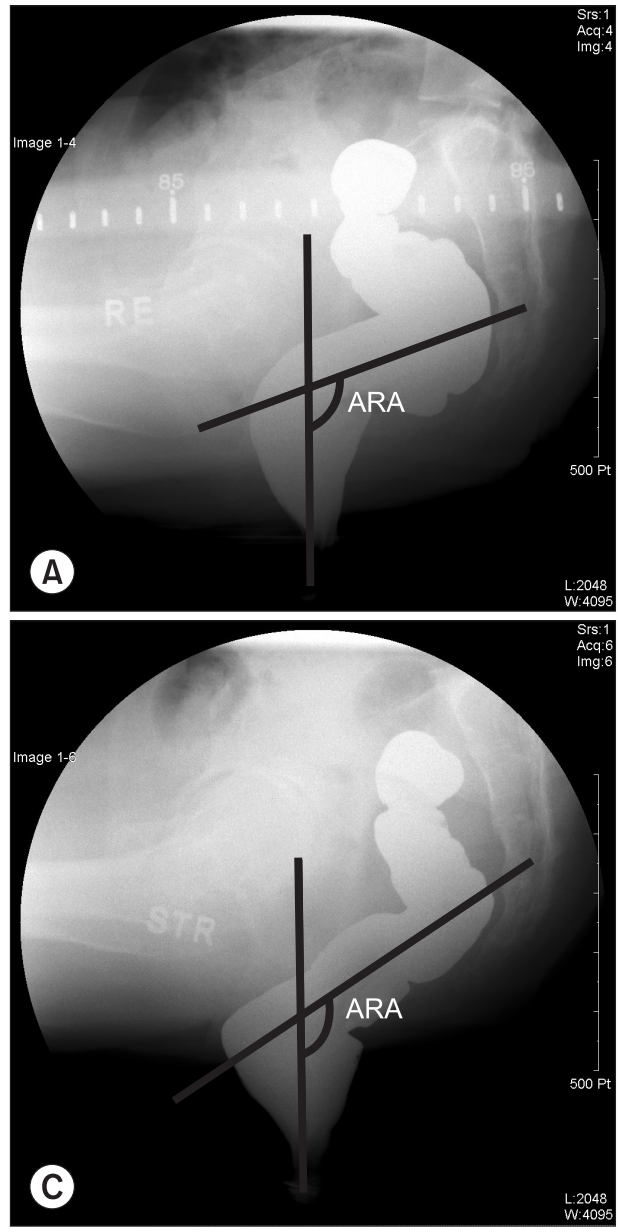

Srs: 1
Acc:
Img: 6

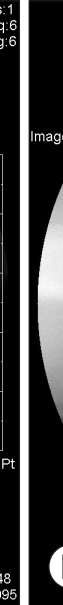

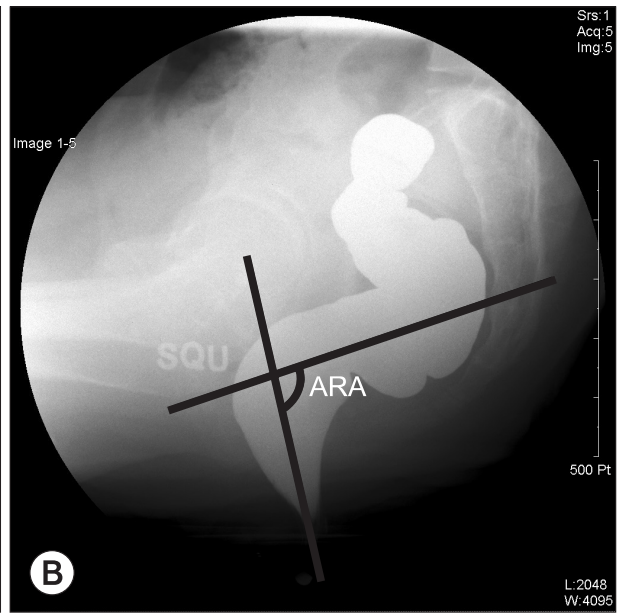

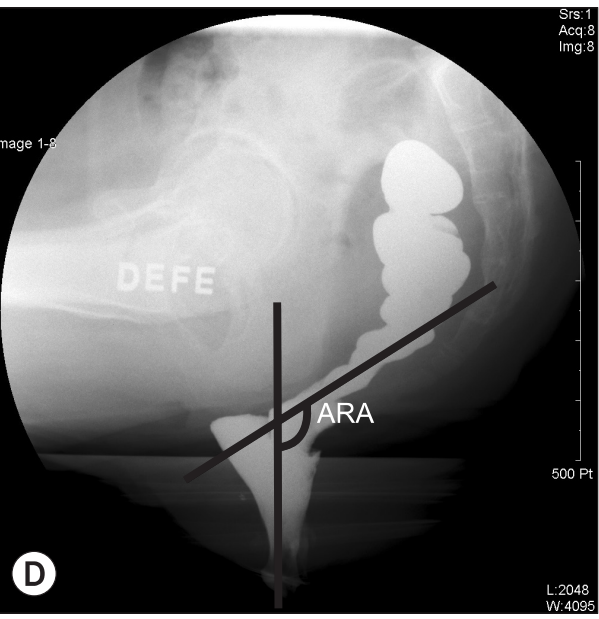

Fig. 3. Defecogram. Defecogram at rest (A), squeezing (B), straining (C) and defecation (D). The anorectal angle (ARA) is measured between the anal canal and the central longitudinal axis of the lower rectum. ARA decreased when squeezing (B), however not enough to reach normal values. Also, a tendency for proctocolic intussusceptions was observed when defecating (D). 
initiated due to the request of the patient.

The patient continued to experience dyschezia, but voiding difficulties improved and self voiding urine was $300-400 \mathrm{ml}$ with residual urine of $50-70 \mathrm{ml}$. The patient decided to follow up on an outpatient basis while continuing treatment options like biofeedback physiotherapy, but she has not fully recovered currently at 7 month.

\section{Discussion}

An epidural block using local anesthetics is commonly used for analgesia for pain relief of the vaginal delivery process. And when complications arise, epidural blocks are rarely the cause [2,3]. According to Holdcroft et al. [4], neurological complications for those that receive an epidural block or anesthesia occur at a low rate of 1 out of 13,007 patients, while those caused by the obstetric procedure itself or other causes can occur at rates of 4-6 times that.

If neurological complications from an epidural block occur, it can present as paresthesia and a reduction of muscular strength. Nerve damage can be caused by the Tuohy needle, the catheter, spinal cord ischemia, accidental injection of neurotoxic agents, infection or injection of local anesthetics into the epidural space. Also neurological complications from an epidural hematoma can occur, but such cases are rare at a rate of $1: 150,000$ [5]. Most hematoma cases occur when a patient has problems with coagulation, therefore preeclamptic patients with a risk of coagulopathy have a higher risk. In these patients the time of injection and removal of the catheter is very important. Also, 24 hours following a catheter removal, a thorough neurological examination should be taken. If complications arise, sharp leg and back pains can occur followed by a loss of sensation and strength in both extremities, incontinence, lack of tension of the sphincter ani, and a loss of motor reflexes. If hematoma is suspected, computer tomography or MRI should be taken and after a hematoma diagnosis, surgical decompression is needed to commence in 6 hours [6].

The epidural needle can enter a blood vessel, pierce the intervertebral foramen or get tangled in the spinal nerve root and cause complications [7,8]. If the spinal nerve root is injured during the procedure, in most cases, unconscious leg movement and radiculopathy occurs, in which the procedure should be immediately stopped and the placement of the needle or catheter should be properly adjusted. Complications resulting from improper epidural catheter placement can be more severe than that of direct damage from a needle or drugs. Recovery from injury of the nerve root of the spine takes a long time, usually months after damage, so extra care should be taken.

During the case in question, because there was no pain or paresthesia until the catheter was removed, the likelihood of nerve damage by the Tuohy needle or epidural catheter seems unlikely. Also, no neurological symptoms were observed during the injection of the drugs, so nerve damage from the toxicity of the drugs also seems unlikely.

If neurological complications are suspected, taking a thorough history of the symptoms is vital to the differentiation of the cause of the damage. The onset, area of the symptom, presence of radiating pain or pain during the procedure should be inquired. Also, through physical examination, it should be recognized whether the pain or the neurologic deficit follows the dermatome or the pathways of the peripheral nerves. An EMG helps to figure out the time of injury and area. Because changes in the EMG shows after 2 weeks of the injury, if changes occur in the first week of delivery, it means the mother had neurologic problems before the delivery.

During vaginal delivery, various factors can lead to nerve damage such as improper vacuum or forceps operation, inappropriate position of the mother, and pressure from the fetal head. Examples include: damage to the Lumbar plexus, sacral plexus, femoral nerve, obturator nerve, common peroneal nerve, and tibial nerve are examples. The pudendal nerve can also receive damage during vaginal delivery [9], and denervation of the sphinter ani can cause trouble with defecation $[10,11]$. It is not known if such neurological damage is caused by extension of the pudendal nerve or direct pressure from the fetal head at the small branch of the nerve or neuromuscular junction.

The sacral plexus is derived from the anterior rami of spinal nerves L4, L5, S1, S2, S3, and S4. Each of these anterior rami gives rise to anterior and posterior branches which provide motor and sensory nerves for the posterior thigh, most of the lower leg, the entire foot, and part of the pelvis. If damages to the sacral plexus were to occur, urination and defecation disorders similar to cauda equina syndrome can occur, which is due to damage to the automatic nervous system of the rectum and bladder. The pelvic splanchnic nerve from the ventral roots, through the parasympathetic fiber and pudendal plexus, sends arousal signals to the bladder's destrusor muscle, while it sends repression signals to the internal sphincter muscle of the urethra and the smooth muscle of the rectum. The sensation of pain and expansion from the bladder and lower rectum is sent to the central nervous system, it passes through the pudendal nerve and posterior rami to be terminated at the anterolateral column of the S2-4 spine. The pudendal nerve also includes motor fibers and relays repression or arousal signals to the external sphincter of the urethra and anus.

In this case, urological testing showed a flaccid neurogenic bladder, which was consistent with the T11, T12, L1 and L2 sympathetic nerve signaling the bladder, while S2 through S4 parasympathetic nerve signals were being blocked, causing 
detrusor muscle relaxation, and persistent arousal of the internal sphincter muscle of the urethra. Also severe degradation of the desire to void and defecate showed there may have been problems with parasympathetic signaling between the bladder and rectum. Upon evaluation of the patient's anorectal function, an anorectal angle greater than the normal value and proctocolic intussusception during defecation was observed by defecography. But the diagnostic value of this in terms of neuropathy is controversial [12].

There is no specific way of evaluating pelvic splanchnic nerve damage, while the PNTML test exists for pudendal nerve damage. The PNTML test has its limitations in that even while nerve damage has progressed severely, motion conduction ability can be maintained since motion conduction can occur through the small diameter axon of the pudendal nerve [13]. Therefore even minimal fiber activity can yield normal results. Other testing methods excluding PNTML only examines endorgan functions, and it can be said is a practical and accurate method of measuring pudendal nerve function does not exist [14].

Through a combination of results from physical examinations and consequent EMG, MRI, urodynamic study, anorectal manometry, defecography as well as patient urination/ defecation disorder symptoms, we were able to conclude, the sacral plexus, in which the splanchnic nerve and pudendal nerve originates, was injured.

When a mother experiences neurological symptoms after epidural analgesia for a cesarean section or vaginal delivery, the anesthesiologist is the first to be called. Since neurologic regional anesthesia related complications have a lower incidence than obstetric origins, an anesthesiologist may initially have difficulties handling such cases. But it is important for the anesthesiologist to keep in mind that neurological damage during delivery is most often caused by peripheral neuropathy from the obstetric process. Though rare, neurological consequences can occur from anesthesia, so if neurological complications do occur, even while there was nothing unusual during the procedure, it is important to decide on the proper course of management after a thorough history, physical examination, and diagnostic tests.

\section{References}

1. Wong CA, Scavone BM, Dugan S, Smith JC, Prather H, Ganchiff $\mathrm{JN}$, et al. Incidence of postpartum lumbosacral spine and lower extremity nerve injuries. Obstet Gynecol 2003; 101: 279-88.

2. Loo CC, Dahlgren G, Irestedt L. Neurological complications in obstetric regional anesthesia. Int J Obstet Anesth 2000; 9: 99-124.

3. Moen V, Irestedt L. Neurological complications following central neuraxial blockades in obstetrics. Curr Opin Anaesthesiol 2008; 21: 275-80.

4. Holdcroft A, Gibberd FB, Hargrove RL, Hawkins DF, Dellaportas CI. Neurological complications associated with pregnancy. Br J Anaesth 1995; 75: 522-6.

5. Horlocker TT, Wedel DJ. Anticoagulation and neuraxial block: historical perspective, anesthetic implications, and risk management. Reg Anesth Pain Med 1998; 23(6 Suppl 2): 129-34.

6. Kane RE. Neurologic deficits following epidural or spinal anesthesia. Anesth Analg 1981; 60: 150-61.

7. Pan PH, Bogard TD, Owen MD. Incidence and characteristics of failures in obstetric neuraxial analgesia and anesthesia: a retrospective analysis of 19,259 deliveries. Int J Obstet Anesth 2004; 13: 227-33.

8. Doughty A. A precise method of cannulating the lumbar epidural space. Anaesthesia 1974; 29: 63-5.

9. Snooks SJ, Setchell M, Swash M, Henry MM. Injury to innervation of pelvic floor sphincter musculature in childbirth. Lancet 1984; 2 : 546-50.

10. Snooks SJ, Swash M, Mathers SE, Henry MM. Effect of vaginal delivery on the pelvic floor: a 5-year follow-up. Br J Surg 1990; 77: 1358-60.

11. Beersiek F, Parks AG, Swash M. Pathogenesis of ano-rectal incontinence. A histometric study of the anal sphincter musculature. J Neurol Sci 1979; 42: 111-27.

12. Ferrante SL, Perry RE, Schreiman JS, Cheng SC, Frick MP. The reproducibility of measuring the anorectal angle in defecography. Dis Colon Rectum 1991; 34: 51-5.

13. Jones PN, Lubowski DZ, Swash M, Henry MM. Relation between perineal descent and pudendal nerve damage in idiopathic fecal incontinence. Int J Colorectal Dis 1987; 2: 93-5.

14. Seong MK, Yoo YB, Koh SE, Cho J. Methodology of evaluating the function of pudendal nerve. J Korean Surg Soc 2004; 67: 204-7. 